\title{
Profound Shock after Initiation of Clozapine Therapy
}

\section{Andrew Choi*, Payal Sud and Howard Greller}

Chief Resident, North Shore University, Emergency Department, 300 Community Drive, Manhasset, NY 11030, USA

\section{Introduction}

Clozapine, a tricyclic dibenzodiazepine, is an atypical antipsychotic with dopaminergic, muscarinic, histaminic and serotonergic activity that has been used in the treatment of schizophrenia [1]. Although prescribers commonly recognize the hematologic adverse effect agranulocytosis its cardiac side effects, such as myocarditits, are less often considered [2]. A retrospective analysis of an Australian reporting system of patients with recent initiation of clozapine found an incidence of myocarditis about 1 in 500 young adults [3]. Studies since this publication have demonstrated incidences ranging from $0.015 \%$ to $8.5 \%$. This compares to an estimated incidence of clozapine-induced agranulocytosis of between $3.8 \%$ and $8 \%$ [4]. A case definition for clozapine-related myocarditis used by Ronaldson et al described the syndrome as "any new symptoms of myocarditis within 45 days of commencing clozapine with histologic or clinical signs of cardiac dysfunction with either laboratory or radiographic evidence of myocardial pathology or congestive heart failure in the absence of alternative plausible schizophrenia" [5].

Neuroleptic malignant syndrome (NMS) is a spectrum of symptoms characterized by mental status change, rigidity, fever and dysautonomia in the context of neuroleptic therapy. The incidence of neuroleptic malignant syndrome has been estimated to be between 0.02 and 3\% [6]. Although classically associated with typical neuroleptic agents, NMS has been reported with every class of neuroleptic drugs [7]. The classic tetrad of symptoms that is considered to be the hallmark of this condition (fever, rigidity, mental status change and autonomic instability) [8] is not always present. There is a controversial definition of an atypical NMS that may be present without one or more of the classic tetrad of symptoms but including rigidity [9]. In these cases, it has been suggested that the use of creatine phosphokinase (CPK) elevations can be used as a surrogate marker for NMS. The Levenson diagnostic criteria for NMS include 3 major manifestations of fever, rigidity and elevated creatine phosphokinase level along with 6 minor manifestations including tachycardia, abnormal blood pressure, tachypnea, altered consciousness, diaphoresis and leukocytosis. It was proposed that the presence of all three major criteria, or two major and four minor would indicate a high probability of the presence of NMS. A revised International NMS Consensus Criteria was proposed using a numerical scoring system to give differential weight to various diagnostic criteria. These criteria were developed using an international multispecialty expert panel allowing for a more refined and objective basis for the diagnosis of NMS [10].

Both clozapine-induced myocarditis and NMS are potentially fatal and our case highlights the difficulty and importance of recognition, management and therapeutics.

\section{Case Report}

A 49-year-old man with a history of schizoaffective disorder was admitted to the inpatient psychiatry service from his group home for aggressive behavior and suicidal ideation. Outpatient medications included metoprolol ( $25 \mathrm{mg} /$ day $)$, olanzapine $(20 \mathrm{mg} /$ day $)$, valproic acid $(2000 \mathrm{mg} /$ day) and an albuterol inhaler as needed. After discussing the risks and benefits with the patient, the patient's psychiatrist and his next of kin, a trial of clozapine was started on hospital day 8. The initial dose was $12.5 \mathrm{mg}$ twice daily. This dose was slowly titrated over the next 2 weeks to a dose of $275 \mathrm{mg}$ once daily.

On hospital day 22 the patient began to complain of abdominal pain and was found to be febrile to $102^{\circ} \mathrm{F}$. Laboratory tests revealed an elevated creatinine of $1.7 \mathrm{mg} / \mathrm{dL}, \mathrm{BUN}$ of $32 \mathrm{mg} / \mathrm{dL}, \mathrm{WBC}$ of $8.4 \mathrm{~K} / \mathrm{uL}$ and 112,000 platelets per uL. On hospital day 23, a rapid response was called for hypotension to $70 / 40 \mathrm{mmHg}$ with a temperature of $100.3^{\circ} \mathrm{F}$. The patient became obtunded and was intubated for airway protection and transferred to the intensive care unit. He was started on vancomycin and piperacillin/tazobactam, norepinephrine and a septic workup was begun. At this time, his labs included an elevation in creatine kinase to $1437 \mathrm{U} / \mathrm{L}$ (normal range of $30-200 \mathrm{U} / \mathrm{L}$ ), troponin $\mathrm{T}$ of $0.12 \mathrm{ng} /$ $\mathrm{mL}$ (normal range $<0.06 \mathrm{ng} / \mathrm{mL}$ ) and CKMB of $4.1 \mathrm{ng} / \mathrm{mL}(<6.7 \mathrm{ng} /$ $\mathrm{mL}$ ). There was no documented violent outburst requiring restraints that would have accounted for this rise in CPK. A transthoracic echocardiogram revealed severe left ventricular systolic dysfunction with an estimated ejection fraction of 30-35\%. The patient had no prior history of cardiac dysfunction. EKG showed sinus tachycardia at a rate of $102 \mathrm{bpm}$. A lumbar puncture was performed and CSF analysis revealed no segmented neutrophils or malignant cells, and bacterial blood cultures and viral nasal cultures were negative. Cultures obtained from the blood, urine and CSF grew no organisms. The toxicology service was consulted at this time with concern for possible drugrelated toxicity. It was advised that the clozapine be discontinued and supportive care including vasopressor support as needed be continued as well as benzodiazepine for agitation as needed. By hospital day 24, creatinine kinase levels began to fall and returned to normal by hospital day 26. The patient was weaned off of vasopressor and ventilator support. A repeat transthoracic echocardiogram on hospital day 31 revealed normal left ventricular systolic function. It is of note that while turning the patient in the intensive care unit (ICU), one of the nurses noted a profound rigidity in the patient's lower extremities. This observation was not confirmed by the ICU team or by any consultant teams and no mention of this was found in the chart upon review.

\section{Discussion}

Although clozapine has been used frequently in the treatment of refractory schizophrenia, its use carries a dangerous and potentially lifethreatening side-effect profile. Neuroleptic malignant syndrome and clozapine-induced myocarditis are two such drug-induced syndromes that exemplify life threatening drug-related side-effects. Although the

*Corresponding author: Andrew Choi, Chief Resident, North Shore University, Emergency Department, 300 Community Drive, Manhasset, USA, Tel: 339-9275333; E-mail: andy.choi.md@gmail.com

Received April 15, 2014; Accepted May 12, 2014; Published May 19, 2014

Citation: Choi A, Sud P, Greller H (2014) Profound Shock after Initiation of Clozapine Therapy. Cardiol Pharmacol 3: 118. doi:10.4172/2329-6607.1000118

Copyright: ( 2014 Choi A, et al. This is an open-access article distributed under the terms of the Creative Commons Attribution License, which permits unrestricted use, distribution, and reproduction in any medium, provided the original author and source are credited. 
Citation: Choi A, Sud P, Greller H (2014) Profound Shock after Initiation of Clozapine Therapy. Cardiol Pharmacol 3: 118. doi:10.4172/23296607.1000118

diagnosis of each relies on high clinical suspicion in the context of an offending agent, there is as of yet no definitive gold standard for diagnosis and each is considered a diagnosis of exclusion.

The pathogenesis of clozapine-induced myocarditis remains unknown. It has been hypothesized given the timing from initiation of therapy that an IgE-mediated type I hypersensitivity reaction, direct pro-inflammatory cardiotoxic effect or a type III serum-sickness reaction may be implicated [11]. Similarly, the pathophysiology of NMS remains unclear. The sudden blockade of D2 dopamine receptors has been considered a contributing factor. A proposed pathway involves hypothalamic neuroleptic blockade of thermoregulation causing a higher thermal set point that results in heat production via muscular rigidity. Other studies noted a reduction in 5-hydroxyindoleacetic suggesting a biochemical relationship with serotonin syndrome[9]. This central neurotransmitter blockade theory contrasts with a proposed direct toxic effect of neuroleptics on peripheral skeletal muscle causing higher metabolic demand, rigidity and an elevation in serum CPK [11].

We present a case of a 49-year-old man who after undergoing careful titration of clozapine, presented with a spectrum of symptoms that could have been diagnosed as either NMS or myocarditis. The elevation of cardiac biomarkers and depression of normal cardiac function requiring vasopressor support fits the diagnosis of clozapineinduced myocarditis. However, the fever, mental status change, elevation in creatine kinase and autonomic instability in the absence of confirmed infectious etiology also fit a diagnosis of NMS or atypical NMS. Moreover, case reports exist for NMS associated with cardiomyopathy as the principal autonomic disturbance. The early recognition and treatment of a possible life-threatening drug reaction was essential in guaranteeing a good outcome for the patient.

The FDA Adverse Event Reporting System (FAERS) in the United States and the Adverse Drug Reactions Advisory Committee (ADRAC) in Australia have created infrastructures for reporting adverse reactions to medications. The Australian registry has been instrumental in facilitating research on the frequency and characteristics of some major adverse outcomes, including clozapineinduced myocarditis. However, because reporting of such outcomes is strictly voluntary, the registry has some limitations.

As there are is no single pathognomonic finding on physical exam or a gold standard diagnostic test, the non-specific signs and symptoms often do not help the clinician in making the diagnosis of clozapineinduced myocarditis unless suspicion is high. Similarly, the diagnosis of neuroleptic malignant syndrome often presents a challenge to the clinician. Although the physical finding of muscular rigidity is classic, it is not required. Due to the lack of universally accepted diagnostic criteria, it has been noted that there is a difficulty in distinguishing neuroleptic malignant syndrome from other conditions [11].

Our patient presented with a sudden onset of an alteration in mental status, hypotension and fever with signs of systolic heart failure and an acute rise in CPK. This presentation was consistent with either clozapine-induced myocarditis or atypical NMS, both of which are diagnoses of exclusion. The cornerstone of treatment for both NMS and myocarditis is the removal of the offending agent and aggressive supportive measures including active cooling, hydration, intravenous benzodiazepines and vasopressor support as needed. While it can be argued that the exact classification of the patient's adverse reaction was not obvious, it is clear that high clinical suspicion of an adverse drug reaction to the clozapine was important to remove the offending agent in a timely fashion.

\section{Reference}

1. Ronaldson KJ, Fitzgerald PB, Taylor AJ, Topliss DJ, Wolfe R, et al. ( 2012) Rapid clozapine dose titration and concomitant sodium valproate increase the risk of myocarditis with clozapine: A case-control study. Schizophrenia Research 141:173-178.

2. Merrill DB, Ahmari SE, Bradford JME, Jeffrey AL (2006) Myocarditis during Clozapine Treatment. Am J Psychiatry 163: 204-208.

3. Killian JG, Kerr K, Lawrence C, Celermajer DS (1999) Myocarditis and Cardiomyopathy Associated with Clozapine. The Lancet 354:1841-1845.

4. Cohen D, Bogers JP, van Dijk D, Bakker B, Schulte PF (2012) Beyond White Blood Cell Monitoring: Screening in the initial phase of clozapine therapy. J Clin Psychiatry 73: 1307-1312.

5. Ronaldsona KJ, Fitzgeraldb PB, Taylorc AJ, Toplissd DJ, Rory Wolfea, et al. (2012) Rapid clozapine dose titration and concomitant sodium valproate increase the risk of myocarditis with clozapine: A case-control study. Schizophrenia Research 141: 173-178.

6. Levenson JL (1985) Neuroleptic malignant syndrome. American Journal of Psychiatry. 142:1137-1145.

7. Caroff SN (1992) Neuroleptic malignant syndrome. Medical Clinics of North America. 77:185-202

8. Velamoor VR (1998) Neuroleptic malignant syndrome. Recognition, prevention and management. Drug Safety. 19:73-82.

9. Carroll BT (2009) the problem of atypical neuroleptic malignant syndrome: a case report. Psychiatry. 6:45-47.

10. Buckley, PF (1995) Neuroleptic Malignant Syndrome. Journal of Neurology, Neurosurgery and Psychiatry. 58: 271-273.

11. Oomura M, Terai T, Sueyoshi K, Shigeno K (2004) Reversible cardiomyopathy as the autonomic involvement of neuroleptic malignant syndrome. Internal Medicine 43: 1162-1165. 